\title{
CORRECTIONS
}

\section{Whistleblowing: The price of silence}

In this article by Jonathan Gornall (BMJ 2009;339:b3202,

doi:10.1136/bmj.b3202) we should have included the following

Cite this as: $B M J$ 2011;342:d3567

statement: "The $B M J$ thanks Andrew Bousfield for alerting us

to this story and for help in providing necessary documentation." 